\title{
Avaliação do Grau e da Extensão das Alterações Térmicas Produzidas pela Cirurgia de Alta Freqüência no Colo Uterino
}

\author{
Evaluation of the Intensity and the Extension of Thermal Alterations Produced by High \\ Frequency Surgery in the Uterine Cervix
}

\begin{abstract}
Nabiha Saadi Abrahão Taha, José Focchi, Julisa Chamorro Lascalas Ribalta, João Norberto Stávale Gerson Botacini das Dôres, Geraldo Rodrigues de Lima
\end{abstract}

\section{RESUM0}

Objetivo: estudar a ocorrência de dano térmico tissular nos espécimes excisados por cirurgia de alta freqüencia e avaliar qualitativa e quantitativamente o dano térmico sobre o epitélio ectocervical e o endocervical e a provável influência do estado menstrual sobre sua gênese. Método: estudo prospectivo de 100 pacientes com lesão cervical intra-epitelial de alto grau. O dano térmico encontrado foi subdividido em três graus, leve, moderado e grave, de acordo com os critérios de Messing et al. ${ }^{1}$

Resultado: a análise estatística permitiu avaliar que o dano térmico ocorreu em todos os casos, porém em 91\% das vezes ele foi insignificante, permitindo a correta avaliação histopatológica. A extensão da alteração térmica tecidual sobre o epitélio endocervical foi de $271,6 \mu$, ao passo que a extensão no epitélio ectocervical foi de 254,8 $\mu$, mostrando desta forma que a extensão do dano térmico é significantemente maior no epitélio endocervical. Das 100 pacientes avaliadas, 80 estavam no menacme e 20 na menopausa. O grau e a extensão do dano térmico tissular não variaram com o estado menstrual.

Conclusão: não se observou qualquer diferença significativa na avaliação tanto do ponto de vista qualitativo quanto quantitativo. Por outro lado, evidenciou-se que na prática cotidiana não há necessidade de se fazer a mensuração do dano térmico.

PALAVRAS-CHAVE: Colo uterino: lesões pré-neoplásicas. Conização. Cirurgia de alta freqüência.

\section{Introdução}

O carcinoma in situ do colo uterino, pela sua importância e freqüência, tem sido estudado desde $1910^{2-4}$. Sua gênese está associada ao papilomavirus humano (HPV) e a fatores socioeconômicos e comportamentais ${ }^{5-7}$. Uma vez encontrada a lesão, a biópsia colposcopicamente dirigida e o estudo histopatológico são utilizados para firmar o diagnóstico, excluir doença invasiva e ajudar na decisão terapêutica. Dentre as alternativas terapêuticas citam-se aquelas que destroem

Departamento de Ginecologia da Universidade Federal de São Paulo - Escola Paulista de Medicina

Correspondência: Nabiha Saadi Abrahão Taha

Rua Borges Lagoa n.1231 cj.62 - Vila Clementino

04038-034 - São Paulo - SP

Tel: (11) 5084-0100 - Fax: (11) 5574-0658

e-mail:taha@uol.com.br o tecido, podendo ser realizadas pela diatermocoagulação, crioterapia ou com laser, e as excisionais, como a conização com bisturi ou com o laser ${ }^{8-11}$

Os procedimentos de destruição tissular são facilmente realizados e apresentam baixa morbidade e alta taxa de sucesso terapêutico. Entretanto, somente podem ser indicados quando a atipia colposcópica é totalmente visivel e a doença invasiva foi definitivamente afastada. Por sua vez, os métodos excisionais fornecem quantidade de material suficiente para o correto diagnóstico histopatológico. A excisão do colo por meio de uma alça, utilizada tanto para o diagnóstico como para o tratamento das lesões pré-neoplásicas cervicais, tem sido utilizada com sucesso nos últimos 10 anos, sendo a primeira opção terapêutica na maioria dos serviços de patologia cervical. Recebe várias denominações, LLETZ, LEEP, radiocirurgia, e 
é conhecida em nosso meio como CAF (cirurgia de alta freqüência) ${ }^{4,12,13}$. Todavia, o principal ponto de discussão sobre essa técnica diz respeito ao possivel dano térmico tissular produzido pelas altas temperaturas no leito de ressecção, o que poderia dificultar a avaliação histológica precisa do espécime. A literatura apresenta controvérsias quanto aos danos térmicos induzidos pela CAF. Montz et al. ${ }^{14}$ evidenciaram que causa alta taxa de destruição tissular nas margens cirúrgicas, com relativa limitação para correta interpretação histopatológica. Entretanto, vários outros estudos afirmam que os espécimes excisados pela CAF têm margem de ressecção satisfatória, permitindo avaliação adequada ${ }^{15-17}$.

A fim de aquilatar a real incidência e extensão do dano térmico ocasionado pela CAF, realizamos o presente estudo, no qual somente um cirurgião efetuou os procedimentos, com o mesmo tipo de equipamento. Avaliou-se também a possivel influência do estado menstrual sobre a qualidade dos espécimes.

\section{Pacientes e Métodos}

Foram estudados prospectivamente 100 espécimes de conização de colo uterino obtidos por CAF realizada em pacientes não-grávidas e nãoportadoras de discrasias sanguíneas. Todas apresentavam diagnóstico citológico, colposcópico e histopatológico de lesão intra-epitelial de alto grau (LIEAG). A média de idade das pacientes foi de 42,5 anos (intervalo de variação de 19 a 74). Oitenta pacientes estavam no menacme. O protocolo do estudo foi aprovado pela comissão de ética de pesquisa e de assistência do Hospital São Paulo.

A localização da atipia colposcópica determinou o tipo de procedimento cirúrgico. Nos casos com atipia ectocervical com visão completa da zona de transformação, realizou-se a excisão ampla da mesma. Na presença de lesões endocervicais ou quando a junção escamo-colunar não era visivel efetuou-se a conização, segundo técnica descrita por Wright et al. ${ }^{17}$ e Dôres ${ }^{12}$. O aparelho utilizado era de circuito elétrico, microprocessado, digital, com conexões-padrão, controle manual, possuindo três tipos de ondas e função stand by.

Os seguintes parâmetros foram avaliados: gradação qualitativa e quantitativa do dano térmico por meio de sua medida máxima em micra $(\mu)$ sobre o epitélio ectocervical e endocervical. As alterações térmicas tissulares qualitativas foram distribuídas em três graus: leve (I), moderado (II) e grave (III), de acordo com os critérios de Messing et al. ${ }^{1}$. No efeito grau I observou-se leve alteração térmica que permitiu, sem dificuldade, a avaliação histopatológica, com identificação precisa das margens cirúrgicas e do grau de neoplasia. Considerou-se como grau II, quando da presença de moderada alteração térmica que ocasionava relativa dificuldade tanto na correta interpretação do grau da neoplasia, quanto no real comprometimento das margens de ressecção. Finalmente, quando os espécimes apresentavam intenso dano térmico ou perda de epitélio que não permitia a avaliação histopatológica tanto do grau da neoplasia quanto da margem cirúrgica as lesões foram classificadas como grau III.

A medida da extensão das alterações térmicas epiteliais e no tecido conjuntivo foram tomadas utilizando-se o sistema computadorizado de análise digital de imagem, Kontron Imaging System KS 300 - Carl-Zeiss. No estudo estatístico, para a comparação entre dois grupos, utilizou-se o teste de Mann-Whitney para variáveis em que não se conhecia o tipo de distribuição e o teste do $\chi^{2}$, para variáveis previamente definidas. Utilizouse o teste de Wilcoxon para se verificar a possivel diferença entre as medidas médias das margens de ressecção ectocervical e endocervical.

\section{Resultados}

Analisando-se os resultados qualitativos do dano térmico, observa-se, na Tabela 1, que eles estão presentes em todos os casos submetidos a esse procedimento, sendo que o de grau I, o mais freqüente, ocorreu em $91 \%$ dos casos. Por sua vez, o grau II esteve presente em $8 \%$ dos espécimes e o de grau III, aquele que impede a total leitura das lâminas, foi verificado em apenas 1\% das vezes.

Tabela 1 - Distribuição do grau do efeito térmico em 100 espécimes.

\begin{tabular}{ccccccccc}
\hline & \multicolumn{2}{c}{ Grau I } & \multicolumn{2}{c}{ Grau II } & \multicolumn{2}{c}{ Grau III } & \multicolumn{2}{c}{ Total } \\
& $\mathbf{n}$ & $\%$ & $\mathbf{n}$ & $\%$ & $\mathbf{n}$ & $\%$ & $\mathbf{n}$ & $\%$ \\
\hline No espécimes & 91 & 91 & 8 & 8 & 1 & 1 & 100 & 100 \\
\hline
\end{tabular}

Como pode ser observado na Tabela 2, a extensão do dano térmico no epitélio endocervical é significantemente maior e a medida máxima de extensão observada foi ao redor de $540 \mu$.

A avaliação qualitativa do efeito térmico nos grupos menopausa e não-menopausa, Tabela 3 , 
não mostrou qualquer diferença significativa. Da mesma forma, a extensão da alteração térmica também foi a mesma nos dois grupos.

Tabela 2 - Correlação entre as medidas da margem ectocervical e endocervical em relação ao grau I. Intervalo e média das medidas máximas.

\begin{tabular}{lcc}
\hline & Ectocervical & Endocervical \\
\hline Intervalo & $30,05-485,68$ & $54,05-538,49$ \\
Média & 254,88 & 271,67 \\
\hline$p=0,0972$ & &
\end{tabular}

Tabela 3 - Correlação entre estado menstrual e dano térmico.

\begin{tabular}{|c|c|c|c|c|c|}
\hline $\begin{array}{l}\text { Estado } \\
\text { menstrual }\end{array}$ & GI(\%) & $G \|(\%)$ & GIII(\%) & $\begin{array}{c}\text { Ectocervical } \\
\text { (média - } \mu \text { ) }\end{array}$ & $\begin{array}{l}\text { Endocervical } \\
\text { (média - } \mu \text { ) }\end{array}$ \\
\hline Menacme & 91,3 & 7,5 & 0,1 & 254,88 & 251,80 \\
\hline Menopausa & 90,0 & 10,0 & 0,0 & 246,73 & 267,36 \\
\hline Total & 91,0 & 8,0 & 1,0 & & \\
\hline
\end{tabular}

\section{Discussão}

Analisando-se os resultados qualitativos do dano térmico, conclui-se que eles estão presentes em todos os casos submetidos a esse procedimento, sendo que o de grau I, o mais freqüente, ocorreu em $91 \%$ dos casos. Por sua vez, o grau II esteve presente em $8 \%$ dos espécimes e o de grau III foi verificado em apenas $1 \%$ das vezes. Esses resultados são concordantes com a maioria dos autores, que mostraram índices variando de 0,5 a $3,3 \%$ de dano tissular severo ${ }^{18-20}$.

Em poucos estudos, o dano tissular grau III é observado em percentagens altas, variando de 26 a $48 \%{ }^{1,14,21,22}$. Segundo Montz et al. ${ }^{13}$, esses valores discrepantes podem ser creditados à baixa freqüência de onda utilizada por alguns autores. Quando se empregam ondas com freqüência igual ou menor a $550 \mathrm{kHz}$, elas são insuficientes para produzir a volatilização celular em tempo hábil, acarretando maior aquecimento e destruição tecidual ${ }^{23}$. É preceito técnico que os geradores utilizados para essa cirurgia devem, obrigatoriamente, produzir freqüência de onda maior que dois $\mathrm{MHz}$. Por isso, utilizando-se este tipo de gerador, pôde-se aqui verificar que a grande maioria dos espécimes não apresentou qualquer dúvida quanto a sua avaliação histopatológica.

$\mathrm{Na}$ definição qualitativa dos graus de alteração térmica II e III o ginecologista não terá certeza do diagnóstico final. Em termos práticos de seguimento das pacientes em cujos cones se observam tais fenômenos, a conduta portanto será a mesma e implicará cuidados redobrados no acompanhamento dessas mulheres ${ }^{24}$. Por se tratar de eventualidades pouco freqüentes, deter-nosemos na análise detalhada da extensão do dano térmico nos casos onde, qualitativamente, se mostrou como grau I.

No que diz respeito aos tecidos excisados por ocasião da conização cervical, deve-se ter em mente que a alça está transpondo dois epitélios morfologicamente distintos, o ectocervical e o endocervical. O ectocervical é epitélio malpighiano (pavimentoso, estratificado e não-queratinizado) que se divide em três camadas. A profunda é constituída de uma fileira única de células basais, que se assentam na membrana basal, e quatro a cinco camadas de células parabasais. A intermediária é formada por várias camadas de células que contêm abundante glicogênio no seu citoplasma, e, finalmente, a superficial é constituída também de várias camadas celulares. Todo esse conjunto, com sua morfologia pluriestratificada normal, apresenta espessura de cinco a sete milímetros. Por sua vez, o epitélio endocervical é composto de camada única de células cilíndricas altas com núcleos basais, bem menos espesso ${ }^{25}$.

Dessa forma, é lícito supor que o epitélio endocervical, por ser mais frágil, seria mais susceptivel ao dano térmico. Por isso, na mesma peça cirúrgica, fez-se a correlação quantitativa das alterações térmicas que ocorreram distintamente nesses dois epitélios. De fato, essa assertiva foi verificada e a extensão do dano térmico no epitélio endocervical é significantemente maior. A medida máxima de extensão observada foi próxima de $540 \mu$, e isto permite afirmar que a temperatura gerada no leito de ressecção produz seus efeitos até esta distância. Em decorrência do fato, é lícito afirmar que, por ocasião da excisão, principalmente no epitélio endocervical, a alça deve obrigatoriamente passar a pelo menos $1 \mathrm{~mm}$ da margem externa da lesão. Além do mais, este fato reforça a necessidade de o procedimento ser realizado por colposcopista capacitado a determinar a real extensão da atipia colposcópica ${ }^{20}$. Com estes cuidados, a possibilidade de que o dano térmico atinja o tecido neoplásico, impossibilitando a avaliação histopatológica, fica minimizada.

A suposição de que a maior atrofia e o menor teor de água do colo uterino das mulheres menopausadas pudessem levar a danos térmicos tissulares de maior monta, conforme afirmam Wright et al. ${ }^{17}$, não foi confirmada. A avaliação qualitativa do efeito térmico nos grupos menopausa e não-menopausa não mostrou qualquer diferença estatística. Da mesma forma, a extensão 
da alteração térmica também foi a mesma nos dois grupos. Pelo exposto, pode-se deduzir que a simples referência no laudo histopatológico da existência de efeito térmico não tem qualquer valor. O importante é saber se ele prejudica o estudo histopatológico e, conseqüentemente, o diagnóstico definitivo da doença que a paciente apresenta.

Da mesma forma, não há necessidade de medir, no dia-a-dia, a extensão das alterações epiteliais, visto que em todos os graus de comprometimento tissular as medidas máximas foram similares. Como todos os espécimes apresentam dano térmico, o aparecimento dessas alterações se deve a outros fatores, que não o tipo do epitélio e o estado menstrual. De fato, em outros estu$\operatorname{dos}^{1,14,21,22}$, essas ocorrências são atribuídas ao uso de alta potência de corte, tipo de onda, movimento muito vagaroso da alça, parada do procedimento durante a excisão, pressão do eletrodo sobre o tecido e utilização de eletrodos não-apropriados ou então carbonizados. Na verdade, erros técnicos do operador $^{13,18}$.

Portanto, é de vital importância, nesse simples método de tratar tão freqüente doença, a habilidade do cirurgião, a correta escolha e configuração do gerador de alta freqüência, como também a seleção de alças de ressecção adequadas e de boa qualidade ${ }^{19}$.

\section{SUMMARY}

Purpose: to evaluate the incidence of thermal damage to the specimens excised through large loop excision of the transformation zone (LLETZ) and to determine qualitatively and quantitatively the thermal injury to the ectocervical and endocervical epithelia as well as the influence of the menstrual phase on such process.

Methods: we performed a prospective study of 100 patients with high-grade squamous intraepithelial lesions (HGSIL). Thermal damage was subdivided into three grades according to Messing et al .

Results: thermal injury occurred in all the cases, however, through statistical analysis we found that in $91 \%$ of the cases it was insignificant, thus leading to a precise histological evaluation, hence to measure the thermal injury was unnecessary. The grade and extent of thermal damage in excised specimens using LLETZ had no relation to the menstrual phase. The extension of thermal tissue alteration in the endocervical epithelia was 271,6 $\mu$ while the extension in ectocervical epithelia was $254,8 \mu$, showing that the extension of thermal damage is significantly higher in endocervical epithelia. Of the one hundred patients, 80 were in menacme and 20 in menopause correlating the grade and extension of thermal damage with the menstrual state. Conclusion: there was no significant difference in both qualitative and quantitative evaluations. There is no need to measure the thermal damage.

KEY WORDS: Cervix: neoplastic lesions. Conization. LLETZ.

\section{Referências}

1. Messing MJ, Otken L, King LA, Gallup DG. Large loop excision of the transformation zone (LLETZ): a pathologic evaluation. Gynecol Oncol 1994; 52:207-11.

2. Rubin IC. Pathological diagnosis of incipient carcinoma of the uterus. Am J Obstet Gynecol 1972; 114:1097.

3. Reagan JW, Seidemann IL, Saracusa Y. Cellular morphology of carcinoma in situ and dysplasia or atypical hyperplasia of uterine cervix. Cancer 1953; 6:224-35.

4. Rattray C, DaCosta V, Chatoor J, Mullings A, Wynter HH. Large loop excision of the transformation zone (LLETZ): an alternative treatment for cervical intraepithelial neoplasia. West Indian Med J 1993; 42:62-4.

5. Brinton LA, Fraumeni JF Jr. Epidemiology of uterine cervical cancer. J Chronic Dis 1986; 39:1051-65.

6. La Vecchia C, Franceschi S, Decarli A, et al. Sexual factors, venereal diseases, and the risk of intraepithelial and invasive cervical neoplasia. Cancer 1986; 58:935-41.

7. Koutsky LA, Holmes KK, Critchlow CW, et al. A cohort study of the risk of cervical intraepithelial neoplasia grade 2 or 3 in relation to papillomavirus infection. N Engl J Med 1992; 327:1272-8.

8. Richart RM, Sciarra JJ. Treatment of cervical dysplasia by outpatient electrocauterization. Am J Obstet Gynecol 1968; 101:200-5.

9. Chanen W, Rome RM. Electrocoagulation diathermy for cervical dysplasia and carcinoma in situ: a 15 year survey. Obstet Gynecol 1983; 61:673-9.

10.Focchi J, Leitzke G, Lima Filho OA. Lesões precursoras do câncer do colo do úterodiagnóstico e tratamento. J Bras Ginecol 1987; 97:299-303.

11.Alves AC, Martins NV, Dôres GB, Simões PM, Souza RC, Rodrigues de Lima G. Neoplasias malignas do colo uterino. In: Rodrigues de Lima G, Martins NV. Oncoginecologia. $1^{\mathrm{a}}$ ed. São Paulo: Atheneu; 1992. p. 105-21. 
12.Prendiville $\mathrm{W}$, Davies R, Berry PJ. A low voltage diathermy loop for taking cervical biopsies: a qualitative comparison with punch biopsy forceps. Br J Obstet Gynaecol 1986; 93:773-6.

13. Dôres GB. Cirurgia de alta freqüência no tratamento da Neoplasia Intra- epitelial Grau III do colo uterino [tese]. São Paulo: Universidade Federal de São Paulo; 1996.

14. Montz FJ, Holschneider $\mathrm{CH}$, Thompson LD. Large loop excision of the transformation zone: effect on the pathologic interpretation of ressection margins. Obstet Gynecol 1993; 81:976-82.

15.Bigrigg MA, Haffenden DK, Sheehan AL, Codling BW, Read MD. Efficacy and safety of large-loop excision of the transformation zone. Lancet 1994; 343:32-4.

16.Prendiville W. Large loop excision of the transformation zone. Clin Obstet Gynecol 1995; 38:622-39.

17.Fanjul R, Pintado S, Velasco J. Lesiones condilomatosas de cérvix uterino: conización LLETZ. Rev Med Univ Navarra 1995; 39:6-10.

18. Wright TC Jr, Gagnon S, Richart RM, Ferenczy A. Treatment of cervical intraepithelial neoplasia using the loop electrosurgical excision procedure. Obstet Gynecol 1992; 79:173-8.

19.Minh HN, Smadja A, Orcel SL. Altérations tissulares déterminées par l'anse diathermique. Rev Fr Gynécol Obstét 1987; 82:563-5.

20.Spitzer M, Chernys AE, Seltzer VL. The use of large loop excision of the transformation zone in an inner-city population Obstet Gynecol 1993; $82: 731-5$

21.Krebs HB, Pastore L, Helmkamp F. Loop electrosurgical excision procedures for cervical dysplasia: Experience in a community hospital. Am J Obstet Gynecol 1993; 169:289-95.

22. Mathevet P, Dargent D, Roy M, Beau G. A randomized prospective study comparing three techniques of conization: cold knife, laser and LEEP. Gynecol Oncol 1994; 54:175-9.

23.Turner RJ, Cohen RA, Voet RL, Stephens SR, Weinstein SA. Analysis of tissues margins of cone biopsy specimens obtained with cold knife, $\mathrm{CO}_{2}$ and $\mathrm{Nd}$ :YAG lasers and radiofrequency surgical unit. J Reprod Med 1992; 37:607-10.

24.Gardeil F, Barry-Walsh C, Prendiville W, Clinch J, Turner MJ. Persistent intraepithelial neoplasia after excision for cervical intraepithelial neoplasia grade III. Obstet Gynecol 1997; 89:41922.

25.Stávale JN. Anatomia patológica do câncer do colo do útero In: Abrão FS, editor. Tratado de Oncologia Genital e Mamária. 1ª ed. São Paulo: Roca; 1995. p.282-92.

\title{
COMUNICADO AOS ASSOCIADOS E LEITORES
}

\section{Para informações e consultas temos à disposição quatro endereços eletrônicos:}

\author{
febrasgopresiden@uol.com.br \\ secretaria_executiva@febrasgo.org.br \\ publicacoes@febrasgo.org.br \\ tego_habilitacao@febrasgo.org.br
} Home page: www.febrasgo.org.br 